\title{
"Alien hand" and loss of bimanual coordination after dominant anterior cerebral artery territory infarction
}

\author{
A W McNABB, W M CARROLL, F L MASTAGLIA
}

From the Department of Neurology and University Department of Medicine, Queen Elizabeth II Medical Centre and Royal Perth Rehabilitation Hospital, Perth, Western Australia

SUMMARY Three patients with dominant anterior cerebral artery territory infarction demonstrated a severe disturbance of upper limb motor control with impaired bimanual coordination, the "alien hand" sign, and intermanual conflict, in addition to signs of callosal interruption and a transcortical motor aphasia. Recordings of movement-related potentials in one patient showed an attenuated Bereitschaftspotential and a greater asymmetry of the NS' component of the premotor negativity with left finger than with right finger movement. The impairment of bimanual motor control and associated abnormal motor behaviour of the right hand in these cases are postulated to be due to involvement of the supplementary motor area and related areas of the medial frontal cortex.

The anterior cerebral artery supplies the rostral sensorimotor cortex and the anterior two-thirds of the corpus callosum by way of its calloso-marginal and pericallosal branches. Proximal occlusion of the anterior cerebral artery may not only damage the motor cortex, resulting in weakness of the contralateral limbs, but may also produce a number of other abnormal motor phenomena. These include compulsive movements of a reflex nature, described as forced grasping and groping, and a grasp reflex. ${ }^{1}$ Abnormal motor behaviour of a more complex and semipurposive nature may also occur in the contralateral upper limb and has been described as the alien hand sign. ${ }^{2}$ In extreme cases, where one hand acts at cross-purposes to the other, the term intermanual conflict has been used to describe this behaviour. Anterior cerebral artery occlusion may also disrupt interhemispheric connections. If the dominant artery is occluded the non-dominant hemisphere is disconnected from centres concerned with the organisation of speech and skilled motor activity in the dominant hemisphere resulting in agraphia and apraxia in the ipsilateral upper limb. ${ }^{45}$ The combination of these effects produces a unique and disabling disturbance of upper limb motor control.

Address for reprint requests: Professor F L Mastaglia, University Department of Medicine, Queen Elizabeth II Medical Centre, Nedlands WA 6009. Australia.

Received 24 April 1987 and in revised form 19 August 1987 Accepted 29 August 1987
We describe three patients with infarction in the dominant anterior cerebral artery territory who dem onstrated the alien hand sign and intermanual conflice as well as marked impairment of bimanual coordio nation as part of their neurological syndrome. It is postulated that these abnormalities of motor behave iour resulted from damage to the supplementar motor area and related areas in the medial frontat cortex.

\section{Case reports}

Case 1

A 75 year old dextral woman was admitted to a rehabilitation hospital 6 weeks after the sudden onset of a dense right hemiplegia and mutism. Her initial illness had been complicated by urinary and bowel frequency, urgency and incontinence. A past history of treated hypertension of 10 years, and a transient episode of right hand paraesthesiae 12 months prior to presentation was elicited.

On examination she was found to have a blood pressure of $150 / 85 \mathrm{~mm} \mathrm{Hg}$ and signs of aortic valve sclerosis. She was alert but disinhibited, laughing readily and inappropriately, and cursing in a jocular fashion. There was a minimal right upper motor neuron facial paresis, mild right upper limb weakness (MRC grade 4/5), particularly evident proximally, and severe weakness of the right leg (grade 1-2/5), particularly distally. Right sided hyperreflexia was present. Vision and somatic sensation were normal. Although initially mute, her speech subsequently was soft and dysfluent with abnormal prosody but normal comprehension, repetition and reading. Object naming to visual and tactile presentation in either hand was intact. She had a left dyspraxic agraphia and 
a mild left ideomotor dyspraxia was also evident with more complex tasks. Bimanual coordination was markedly impaired; for example she was unable to use simultaneously a knife and fork when eating, or to do up buttons when dressing. A number of other abnormal motor phenomena were also noted. On the left side, semi-coordinated movements of the hand or arm would occur whilst speaking or if she was distracted by other tasks. On the right side, a tonic palmar grasp reflex was present, and initially this hand showed an uncontrollable tendency to reach out and take hold of objects and then be unable to release them. The patient would repeatedly express astonishment at these actions. At times the right hand interfered with tasks being performed by the left hand, and she attempted to restrain it by wedging it between her legs or by holding or slapping it with the left hand. Attempts to execute regular repetitive self-paced flexion-extension movements of individual fingers of the right hand resulted in errors of timing and sequencing which were not seen in the left hand.

A cranial CT scan showed extensive infarction of the left superior and medial frontal and parietal cortex and of the genu and body of the corpus callosum (fig 1).

Movement-related potentials Potentials (fig 2) were recorded by averaging $1.5 \mathrm{~s}$ epochs of EEG preceding 100 extension movements of the index finger of each hand from electrodes at $\mathrm{C} 3, \mathrm{Cz}$ and $\mathrm{C} 4$ (10-20 system) referenced to linked-ears. ${ }^{6}$ With movement of the left index finger the negativity preceding movement onset by $150-250 \mathrm{~ms}$, corresponding to the NS' component of Shibasaki et $\mathrm{al}^{7}$ and the

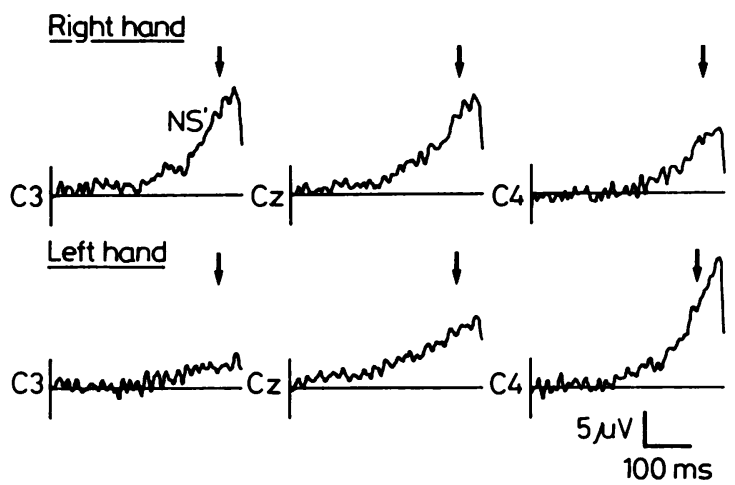

Fig 2 Movement-related potentials associated with right and left finger extension. Note the wider (bilateral) distribution of the NS' component of the premotor negativity with right-sided movements than with left-sided movement, and the attenuation of the preceding Bereitschaftspotential (BP) for both right and left-sided movement. The arrows indicate movement onset which was detected using an infra-red system. ${ }^{6}$

motor potential of Deecke $e t a l^{89}$ and Lee $e t a l,{ }^{10}$ which is thought to represent activity in pyramidal tract neurons in pre- and post-Rolandic areas, ${ }^{10}$ was localised to the (contralateral) C4 electrode site. By contrast, with movement of
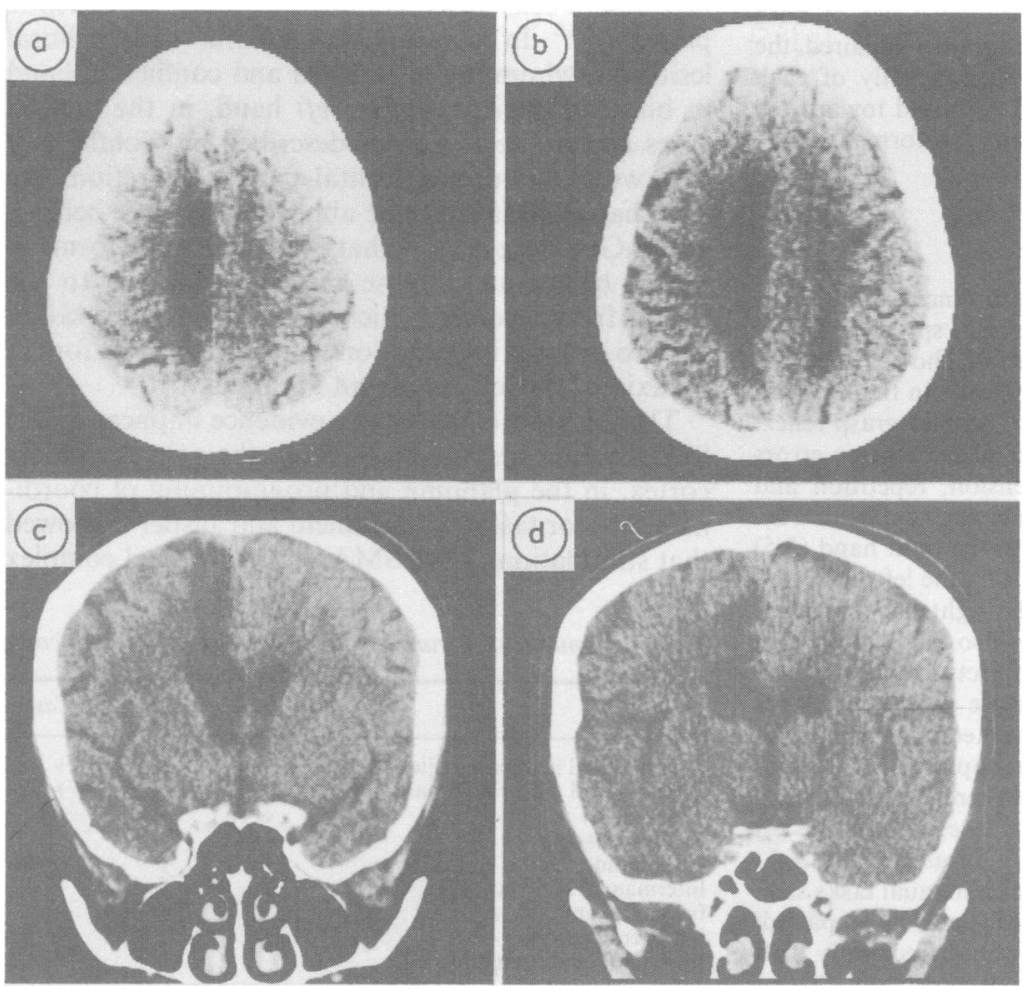

Fig 1 Transverse ( $a$ and $b$ ) and semi-coronal ( $c$ and d) CT scans of Case 1, 2 months after left anterior cerebral artery occlusion showing extensive destruction of the left medial frontal and parietal cortex and corpus callosum. 
the right index finger, this potential was present at each electrode site, although of maximal amplitude contralaterally at C3. The earlier negativity preceding $\mathrm{NS}^{\prime}$ (the Bereitschaftspotential), was poorly developed at all electrode sites for both right and left finger movement. Subcomponents of this earlier negativity as described by Barrett et al ${ }^{11}$ could not be identified.

Case 2 (P.A.)

A 58 year old obese dextral woman was first seen 6 weeks after the sudden onset of right hemiparesis and abnormal speech. There was a past history of endogenous depression, hypertension and a recent myocardial infarction. On examination there was mild right facial and upper limb weakness with a tonic palmar grasp reflex, profound weakness of the right leg, right-sided hyperreflexia, ankle clonus and a Babinski response. Spontaneous speech was hesitant, with word-finding difficulties but normal comprehension, repetition and naming of visually-presented objects. Tactile naming of objects was normal with the right hand $(10 / 10)$ but was severely impaired with the left hand $(0 / 10)$ although in most instances the object could be retrieved from a collection of unrelated items indicating a tactile anomia.

Writing was normal with the right hand while the left hand produced only stereotyped doodling. The left hand was severely dyspraxic making stereotyped waving movements in the air when she was instructed to perform simple or complex movement sequences or to use objects. There was also a constructional apraxia with perseveration when using the right hand. Tasks involving the coordinated use of both hands, such as eating or dressing, were severely impaired, the two hands appearing to act quite independently of each other. At times the right hand would be raised toward the head or would interfere with tasks being performed by the left hand.

\section{Case 3 (R.S.)}

A 68 year old dextral woman was examined some weeks after the acute onset of a right hemiparesis, speech difficulty and urinary incontinence. Examination showed residual right sided weakness which was more severe in the leg, with mild spasticity in the arm and a strong palmar grasp reflex. Her speech was hesitant with occasional paraphasic errors and echolalia but normal comprehension, repetition and visual naming of objects presented in either hemifield. Tactile naming of objects was normal with the right hand $(5 / 5)$ while there was a mild tactile anomia with the left hand $(6 / 8$ correct). Writing was normal with the right hand but was impaired with the left hand which was also mildly dyspraxic when attempting to use common objects or to perform motor sequences. Construction of simple geometric shapes was normal with the right hand but markedly impaired with the left hand. When the patient was attempting to write with her left hand the right hand would frequently be raised and would reach over and attempt to take the pencil from the left hand. The left hand would respond by grasping the right hand to restrain it. The performance of bimanual tasks such as eating or manipulating buttons was severely impaired. During conversation the right hand would frequently drift upwards or make other purposeless movements.

\section{Discussion}

The present cases each displayed the characteristic pattern of limb weakness and associated sphincteric disturbance found after infarction in the territory of the anterior cerebral artery. ${ }^{12}$ In the most recent case (Case 1) the anatomical location of the lesions was confirmed by computed tomography which showed infarction of the medial frontal cortex and corpus callosum. In addition, each patient showed features of a transcortical motor aphasia (table) with reduced, non-fluent spontaneous speech (one patient was mute initially), normal auditory comprehension, reading and repetition, and in one case echolalia (Case 3). This type of aphasia, which has been reported only infrequently in patients with anterior cerebral artery occlusion, ${ }^{13-15}$ has previously been ascribed to lesions involving the supplementary motor area (SMA) or its connections with Broca's area. ${ }^{15-17}$

The lesions responsible for the alien hand and other abnormal motor phenomena have not been well localised. Brion and Jedynak ${ }^{2}$ introduced the concept of the alien hand (la main étrangère) to draw attention to the uncooperative behaviour of the left hand in three patients with tumours of the corpus callosumb Similar phenomena have been described in patient with other lesions of the corpus callosum and for the first few weeks after commissurotomy in epileptic patients. $^{318}$ In contrast to patients with callosaf lesions in whom the alien hand and conflicting han in bimanual tasks was the left hand, in the presene cases and in the two cases described by Goldberg $a l^{19}$ with left medial frontal cortex infarction, the right hand displayed these abnormalities. We believe, as did Goldberg et al, ${ }^{19}$ that these abnormal forms of motor behaviour are the result of damage to the medial frontal cortex, which includes the SMA, rather than to callosal interruption which is the basis for the apraxia of the left hand and agraphia. ${ }^{45}$

There is now considerable evidence implicating the SMA, which lies upstream from the primary motor cortex, in the planning and programming of coordinated motor tasks. ${ }^{2021}$ Penfield and Jasper ${ }^{22}$ showed that stimulation of the SMA in man evoked complex

Table Summary of neurological findings in the present cases

\begin{tabular}{llll}
\hline & Case 1 & Case 2 & Case 3 \\
\hline Contralateral hemiparesis (leg $>$ arm) & + & + & + \\
Transcortical motor aphasia & + & + & + \\
Grasp reflex & + & + & + \\
Forced grasping & + & - & - \\
Alien hand & + & + & + \\
Intermanual conflict & + & + & + \\
Ipsilateral apraxia & + & + & + \\
Ipsilateral agraphia & + & + & + \\
Ipsilateral tactile anomia & - & + & + \\
\hline
\end{tabular}
然 or

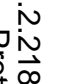

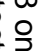
$\vec{r}$ 策

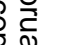
(n)

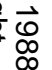


movements of the contralateral upper limb and at times arrest or slowing of movement, as well as vocalisation or speech arrest. Stimulation of the anterior cingulate cortex, which is reciprocally interconnected with the SMA, has also been shown to produce highly integrated forms of motor behaviour. ${ }^{23}$ Regional cerebral blood flow studies in normal subjects performing a sequence of finger movements with one hand showed an increase in blood flow in the SMA bilaterally, even when the subject imagined the unilateral movement sequence without actually making any movement. ${ }^{24}$ Single cell recordings in primates have also shown a relationship between bilateral SMA unit activity and the performance of unilateral learned motor tasks. ${ }^{25}$

Lesions of the dominant SMA in man have been reported to cause mutism, transcortical motor aphasia, forced grasping, groping and a grasp reflex, reduced spontaneous motor activity and impaired bimanual coordination. ${ }^{151626-28}$ Unilateral SMA ablation in monkeys results in a long-lasting deficit in the execution of precision movements of the two hands and marked impairment of the cooperative use of the hands in bimanual tasks, ${ }^{29}$ deficits which are remarkably similar to those observed in the present cases. The alien hand sign and associated abnormal motor behaviour, and the speech disturbance in these cases, may therefore be an expression of impaired programming, execution or inhibition of normal motor subroutines as a result of damage to the SMA or interconnected areas of the dominant medial frontal cortex.

The virtual absence of the Bereitschaftspotential before finger movement in Case 1 is of interest, in view of the postulated origin of this potential from the SMA by some workers. $^{3031}$ If the Bereitschaftspotential does indeed arise from the SMAs, both of which are active during unilateral voluntary movement, it should be attenuated following a destructive lesion of one SMA. However, the significance of this finding in Case 1 is uncertain as, in our experience, it is not always possible to record the potential even in normal subjects and even under optimal recording conditions. Of interest also was the different distribution of the NS' potential with right and left-sided finger movement in this case. This potential is believed to represent activation of pyramidal neurons and is normally distributed bilaterally with a maximal slope contralateral to the side of the movement. $^{711}$ In contrast to normal subjects in whom there is greater asymmetry of NS' $^{\prime}$ for dominant hand movements and a more symmetric distribution with bilateral simultaneous hand movement, ${ }^{32}$ in Case 1 there was a greater bilateral distribution of this potential with right finger movements. It is possible that this difference was due to simultaneous acti- vation of motor centres in both hemispheres because of the difficulty the patient had in making individual finger movements with the right hand without simultaneously activating the left hand.

The authors are grateful to Mr GW Thickbroom who performed the motor potential recordings and to Mrs P McBryde for secretarial assistance.

\section{References}

1 Walton J. Brain's Diseases of the Nervous System, 8th ed. Oxford University Press, 1977.

2 Brion S, Jedynak CP. Trouble du transfert interhémisphérique a propos de trois observations de tumeurs du corps calleux; Le signe de la main étrangère. Rev Neurol (Paris) 1972;126:257-66.

3 Bogen JE. The Callosal Syndrome. In: Heilman KM, Valenstein E, eds. Clinical Neuropsychology. New York: Oxford University Press, 1985:295-338.

4 Liepman H, Maas O. Fall von linksseitiger Agraphie und Apraxie bie rechtsseitiger Lahmung. J Psychol Neurol 1907;10:214-27.

5 Geschwind N. Disconnexion syndromes in animals and man. Brain 1965;88:237-94,585-644.

6 Thickbroom GW, Mastaglia FL, Carroll WM, Davies HD. Cerebral potentials accompanying visually triggered finger movement in man. Electroencephalogr Clin Neurophysiol 1985;62:209-18.

7 Shibasaki H, Barrett G, Halliday E, Halliday AM. Components of the movement-related cortical potential and their scalp topography. Electroencephalogr Clin Neurophysiol 1980;49: 213-26.

8 Deecke L, Scheid P, Kornhuber HH. Distribution of readiness potential, pre-motor positivity and motor potential of the human cerebral cortex preceding voluntary finger movements. Exp Brain Res 1969;7:158-68.

9 Deecke L, Grozinger B, Kornhuber HH. Voluntary finger movement in man: Cerebral potentials and theory. Biol Cybernetics 1976;23:99-119.

10 Lee BI, Luders H, Lesser RP, Dinner DS, Morris HH. Cortical potentials related to voluntary and passive finger movements recorded from subdural electrodes in humans. Ann Neurol 1986;20:32-7.

11 Barrett G, Shibasaki H, Neshige R. Cortical potentials preceding voluntary movement: Evidence for three periods of preparation in man. Electroencephalogr Clin Neurophysiol 1986;63: 327-39.

12 Critchley $M$. The anterior cerebral artery and its syndromes. Brain 1930;53:120-65.

13 Racy A, Jannotta FS, Lehner LH. Aphasia resulting from occlusion of the left anterior cerebral artery. Arch Neurol 1979;36:221-4.

14 Ross ED. Left medial parietal lobe and receptive language functions: Mixed transcortical aphasia after left anterior cerebral artery infarction. Neurology 1980;30:144-51.

15 Alexander MP, Schmidt MA. The aphasic syndrome of stroke in the left anterior cerebral artery territory. Arch Neurol 1980;37:97-100.

16 Freedman M, Alexander MP, Naesarma L. Anatomical basis of transcortical motor aphasia. Neurology 1984;34:409-17.

17 Rubens AB. Transcortical Motor Aphasia. In: Whitaker H, Whitaker A, eds. Studies in Neurolinguistics, Vol l. New York: Academic Press, 1976:293-304.

18 Watson RT, Heilman KM. Callosal apraxia. Brain 1983;106: 391-403.

19 Goldberg G, Meyer NH, Toglia JU. Medial frontal cortex infarction and the alien hand sign. Arch Neurol 1981;38:683-6.

20 Porter R. The brain: seat of motor control. Med J Aust 
1984;140:462-72.

21 Dick JPR, Benecke R, Rothwell JC, Day BL, Marsden CD. Simple and complex movements in a patient with infarction of the right supplementary motor area. Movement Disorders 1986;1:255-66.

22 Penfield W, Jasper HY. Epilepsy and the Functional Anatomy of the Human Brain. Boston: Little Brown and Company, 1954;88:88-102.

23 Talairach J, Bancaud J, Geier S. The cingulate gyrus and human behaviour. Electroencephalogr Clin Neurophysiol 1973;34: 45-52.

24 Roland PE, Larson G, Lassen NA, Skinhog E. Supplementary motor area and other cortical areas in organisation of voluntary movements in man. $J$ Neurophysiol 1980;43:118-36.

25 Brinkman C, Porter R. The supplementary motor area of the monkey. Activity of neurones during performance of motor tasks. J Neurophysiol 1979;42:681-708.

26 Brodal A. Neurological Anatomy in Relation to Clinical Medicine, 2nd ed. Oxford: Oxford University Press 1969:198-9.

27 Damasio AR, van Heusen GW. Structure and function of the supplementary motor area. Neurology 1980;30:359.

28 La Plane D, Talairach J, Meininger V, Bancaud J, Orgogozo JM. Clinical consequences of corticectomies involving the supplementary motor area in man. J Neurol Sci 1977;34:301-14.

29 Brinkman C. Lesions in supplementary motor area interfere with 70 a monkey's performance of a bimanual coordination task. Neurosci Lett 1981;27:267-70.

30 Deecke L, Kornhuber HH. An electrical sign of participation of the mesial "supplementary" motor cortex in human voluntary finger movement. Brain Res 1978;159:473-6.

31 Boschert J, Hink RF, Deecke L. Finger movement versus toe movement related potentials: Further evidence for supple-mentary motor area (SMA) participation prior to voluntary action. Exp Brain Res 1983;52:73-80.

32 Tamas LB, Shibasaki H. Cortical potentials associated with movement. A review. J Clin Neurophysiol 1985;2:157-71. 